\title{
A GEOMETRICALLY ABERRANT BANACH SPACE WITH NORMAL STRUCTURE
}

\author{
J.R. Giles, Brailey Sims and S, Swaminathan
}

\begin{abstract}
An example is given of a Banach space with normal structure which does not satisfy the geometrical conditions commonly expected to be related to normal structure.
\end{abstract}

A Banach space is said to have normal structure if for each nontrivial bounded convex subset $K$ there exists a point $p \in K$ such that $\sup \{\|p-x\|: x \in K\}<\operatorname{diam} K$.

A Banach space is said to have uniformly normal structure if there exists a $0<k<1$ such that for each bounded convex subset $K$ there exists a point $p \in K$ such that

$$
\sup \{\|p-x\|: x \in K\} \leq k \operatorname{diam} K \text {. }
$$

Normal structure was introduced by Brodski i and Milman [2] and has been significant in the development of fixed point theory. A recent survey of results on normal structure has been given by Swaminathan [11].

Considerable research has been directed into finding geometrical conditions which imply normal structure.

A Banach space is said to be uniformly rotund if for any given $\varepsilon>0$ there exists a $\delta>0$ such that, for $x, y,\|x\|=\|y\|=1,\|x-y\|<\varepsilon$

Received 7 August 1984. The research of the third author was supported by NSERC Grant A-5615 as a visiting professor at the University of Newcastle, New South Wales.

Copyright Clearance Centre, Inc. Serial-fee code: 0004-9727/85 $\$$ A2.00 +0.00 . 
when $\|x+y\|>2-\delta ;$ such a space has uniformly normal structure, [5].

A Banach space is said to be uniformly rotund in every direction if for any given $z \neq 0$ and $\varepsilon>0$ there exists a $\delta(\varepsilon, z)>0$ such that $|\lambda|<\varepsilon$ for $x, y,\|x\|=\|y\|=1$ and $x-y=\lambda z$ when $\|x+y\|>2-\delta$; such a space has normal structure, [4], but not necessarily uniformly normal structure, [8].

A Banach space is said to be weakly uniformly Kadec-Klee if there exists an $\varepsilon<1$ and a $\delta>0$ such that for every sequence $\left\{x_{n}\right\}$, $\left\|x_{n}\right\| \leq 1$ which converges weakly to $x$ and $\inf \left\{\left\|x_{n}-x_{m}\right\|: m \neq n\right\} \geq \varepsilon$ we have $\|x\| \leq 1-\delta$. Van Dulst and Sims have recently shown that such a space has weak normal structure, that is, the normal structure property holds for weakly compact convex sets, [12].

A Banach space is said to be localzy uniformly rotund if for any given $x,\|x\|=1$ and $\varepsilon>0$ there exists a $\delta(\varepsilon, x)>0$ such that $\|x-y\|<\varepsilon$ for $\|y\|=1$, when $\|x+y\|>2-\delta$. Smith and Turett have recently provided an example of a reflexive locally uniformiy rotund space which does not have normal structure, [10].

In this paper we give an example of a reflexive Banach space which lacks all of these geometrical properties but which does have normal structure.

In order to produce an example of a discontinuous metric projection Brown devised a geometrically interesting equivalent renorming of Hilbert sequence space $z^{2}$, [3]. Given natural basis $\left\{e_{n}\right\}$ and writing

$$
M \equiv\left\{\left\{\lambda_{n}\right\} \in z^{2}: \lambda_{1}=0\right\}
$$

and

$$
M_{k} \equiv \operatorname{sp}\left\{e_{1}, e_{k}\right\} \text { for } k \geq 3 \text {, }
$$

$i^{2}$ can be given an equivalent rotund norm $\|\cdot\|$ such that its restriction to $M$ remains the original $l^{2}$-norm $\|\cdot\|_{2}$ and its restriction to $M_{k}$ is an $\imath^{p(k)}$-norm where $p(k) \rightarrow \infty$ as $k \rightarrow \infty$. 
Brown's space is not uniformly rotund in every direction. For $x_{k} \equiv\left(e_{1}+e_{k}\right) /\left\|e_{1}+e_{k}\right\|$ and $y_{k}=\left(-e_{1}+e_{k}\right) /\left\|-e_{1}+e_{k}\right\|$,

$$
x_{k}-y_{k}=\frac{2}{2^{1 / p(k)}} \cdot e_{1} \text { and }\left\|x_{k}-y_{k}\right\| \rightarrow 2 \text { as } k \rightarrow \infty
$$

but

$$
x_{k}+y_{k}=\frac{2}{2^{1 / p(k)}} \cdot e_{k} \text { and }\left\|x_{k}+y_{k}\right\| \rightarrow 2 \text { as } k \rightarrow \infty \text {. }
$$

Brown's space is not weakly uniformly Kadec-Klee. For $x_{k} \equiv\left(e_{1}+e_{k}\right) /\left\|e_{1}+e_{k}\right\|$ and any $y \equiv \sum \alpha_{n} e_{n}$,

$$
\begin{aligned}
\left(x_{k}, y\right) & =\frac{1}{2^{1 / p(k)}}\left(\alpha_{1}+\alpha_{k}\right) \\
& \rightarrow \alpha_{1} \text { as } k \rightarrow \infty \\
& =\left(e_{1}, y\right) .
\end{aligned}
$$

So the sequence $\left\{x_{k}\right\}$ converges weakly to $e_{1}$. But

$$
\begin{aligned}
\left\|x_{k}-x_{l}\right\|_{2}^{2} & =\left\|e_{1}\left(\frac{1}{2^{1 / p(k)}}-\frac{1}{2^{1 / p(l)}}\right)+\frac{e_{k}}{2^{1 / p(k)}}-\frac{e_{l}}{2^{1 / p(\eta)}}\right\|_{2}^{2} \\
& =\left(\frac{1}{2^{1 / p(k)}}-\frac{1}{2^{1 / p(l)}}\right)^{2}+\frac{1}{2^{2 / p(k)}}+\frac{1}{2^{2 / p(l)}} \\
& \rightarrow 2 \text { as } k, \imath \rightarrow \infty .
\end{aligned}
$$

However, $(1 / \sqrt{2})\|x\|_{2} \leq\|x\| \leq\|x\|_{2}$ for all $x \in z_{2}$ so

$$
\lim _{k, l \rightarrow \infty} \inf _{k}\left\|x_{k}-x_{\imath}\right\| \geq 1
$$

Therefore, for every $0<\varepsilon<1$,

$$
\lim \inf \left\{\left\|x_{k}-x_{\imath}\right\|: k \neq \imath\right\} \geq \varepsilon ;
$$

but $\left\|e_{1}\right\|=1$.

Brown's space is not locally uniformly rotzond. For $x_{k} \equiv\left(e_{1}+e_{k}\right) /\left\|e_{1}+e_{k}\right\|$, 


$$
\begin{aligned}
\left\|e_{1}+x_{k}\right\| & =\left\|\left(1+\frac{1}{2^{1 / p(k)}}\right) e_{1}+\frac{1}{2^{1 / p(k)}} e_{k}\right\| \\
& =\left(\left(1+\frac{1}{2^{1 / p(k)}}\right)^{p(k)}+\frac{1}{2}\right)^{1 / p(k)} \\
& \rightarrow 2 \text { as } k \rightarrow \infty .
\end{aligned}
$$

But

$$
\begin{aligned}
\left\|e_{1}-x_{k}\right\| & =\left\|\left(1-\frac{1}{2^{1 / p(k)}}\right) e_{1}-\frac{1}{2^{1 / p(k)}} e_{k}\right\| \\
& =\left(\left(1-\frac{1}{2^{1 / p(k)}}\right)^{p(k)}+\frac{1}{2}\right)^{1 / p(k)} \\
& \rightarrow 1 \text { as } k \rightarrow \infty .
\end{aligned}
$$

Nevertheless, as a reflexive Banach space containing a Hilbert subspace of codimension one, Brown's space does have normal structure as is evident from the following general result.

LEMMA. If a Banach space $X$ contains a closed subspace $M$ of finite codimension with uniformly normal stmucture then $X$ has normal structure.

Proof. Since $M$ has uniformly normal structure it is reflexive, [9], and since $X$ contains a reflexive subspace of finite codimension it too is reflexive. Suppose that $X$ does not have normal structure. Then by the characterisation theorem of Brodski $i$ and Mi Iman [2], there exists a weakly compact convex subset $K$ containing a sequence $\left\{x_{n}\right\}$ such that

$$
d\left(x_{n+1}, \operatorname{co}\left\{x_{1}, \ldots, x_{n}\right\}\right) \rightarrow \operatorname{diam} K \text { as } n \rightarrow \infty \text {. }
$$

Subsequences of $\left\{x_{n}\right\}$ satisfy this property so we may, by weak compactness assume that $\left\{x_{n}\right\}$ converges weakly; by translation we may assume that $\left\{x_{n}\right\}$ is weakly convergent to 0 ; by scaling we may assume that diam $K=1$. Consider a linear projection $P$ from $X$ onto $M$. Since $\left\{x_{n}\right\}$ converges weakly to 0 so $\left\{x_{n}-P x_{n}\right\}$ is convergent to 0 in the finite dimensional complement of $M$.

Given $0<k<1$ the constant associated with the uniformly normal structure of $M$, choose $0<\varepsilon<(1-k) / 4(1+k)$. Then there exists a $\nu$ such that 


$$
\begin{aligned}
& \text { An aberrant Banach space } \\
& \left\|x_{n}-P x_{n}\right\|<\varepsilon \text { for all } n>v .
\end{aligned}
$$

Consider $K^{\prime} \equiv \operatorname{co}\left\{x_{n}: n>v\right\}$. Now $K^{\prime}$ has the diametral property that, for any $x \in K^{\prime}$,

$$
\sup \left\{\|x-y\|: y \in K^{\prime}\right\}=\operatorname{diam} K^{\prime}=1 \text {. }
$$

Since $\|x-P x\|<\varepsilon$ for all $x \in K^{\prime}$, it follows that diam $P\left(K^{\prime}\right) \leq 1+2 \varepsilon$. From the uniformly normal structure of $M$ there exists a $p \in K^{\prime}$ such that

$$
\|P p-P x\| \leq k(1+2 \varepsilon) \text {. for all } x \in K^{\prime} .
$$

But then, for all $x \in K^{\prime}$,

$$
\begin{aligned}
\|p-x\| & \leq\|p-P p\|+\|P p-P x\|+\|P x-x\| \\
& \leq 2 \varepsilon+k(1+2 \varepsilon) \\
& <\frac{1}{2}(1+k)<1
\end{aligned}
$$

and this contradicts the diametral property of $K^{\prime}$.

Bernal and Sullivan have recently provided a condition under which an equivalent renorming of Hilbert space has normal structure, [1]. Given a Hilbert space $\left(X,\|\cdot\|_{2}\right)$ and a norm $\|\cdot\|$ on $X$ such that

$$
\frac{1}{\beta}\|x\|_{2} \leq\|x\| \leq\|x\|_{2} \text { for all } x \in X
$$

where $1 \leq \beta<\sqrt{2}$, then the Banach space $(X,\|\cdot\|)$ has normal structure. However, Brown's renorming of Hilbert space has $\beta=\sqrt{2}$ and is therefore an example which shows that, for equivalent renormings of Hilbert space the Bernal-Sullivan condition is not necessary for normal structure.

As a reflexive Banach space containing a closed subspace with discontinuous metric projection it can be deduced indirectly from Fan and Glicksberg [6] that Brown's space lacks a variety of geometrical properties. Brown's space has also been used by Giles [7] to demonstrate the relationship between geometrical properties used by vlasov in the convexity of the Chebychev set problem. 


\section{References}

[1] Javier Bernal and Francis Sullivan, "Banach spaces that have normal structure and are isomorphic to a Hilbert space", Proc. Amer. Math. Soc. 90 (1984), 550-554.

[2] M.S. Brodski $i$ and D.P. Mi Iman, "On the centre of a convex set", Dokl. Akad. Nauk SSSR 59 (1948), 837-840.

[3] A.L. Brown, "A rotund reflexive space having a subspace of codimension two with a discontinuous metric projection", Michigan Math. J. 21 (1974), 145-151.

[4] M.M. Day, R.C. James and S. Swaminathan, "Normed linear spaces that are uniformly convex in every direction", Canad. J. Math. 23 (1971), 1051-1059.

[5] M. Edelstein, "A theorem on fixed points under isometries", Amer. Math. Monthly 70 (1963), 298-300.

[6] Ky Fan and Irving Glicksberg, "Some geometric properties of the spheres in a normed linear space", Duke Math. J. 25 (1958), 553-568.

[7] J.R. Giles, "Uniformly weak differentiability of the norm and a condition of Vlasov", J. Austral. Math. Soc. Ser. A 21 (1976), 393-409.

[8] A.A. Gillespie and B.B. Williams, "Fixed point theorem for nonexpansive mappings on Banach spaces with uniformly normal structure", Applicable Anal. 9 (1979), 121-124.

[9] E. Maluta, "Uniformly normal structure and related coefficients", Pacific J. Math. (to appear).

[10] Mark A. Smith and Barry Turett, "A reflexive LUR Banach space that lacks normal structure", preprint.

[11] S. Swaminathan, "Normal structure in Banach spaces and its generalisations", Contemp. Math. 18 (1983), 101-215. 
[12] D. Van Dulst and Brailey Sims, "Fixed points of non-expansive mappings and Chebychev centres in Banach spaces with norms of type (KK)", Banach space theory and its applications, Bucharest 1981, 91-95 (Lecture Notes in Mathematics, 991. SpringerVerlag, Berlin, Heidelberg, New York, 1983).

Department of Mathematics,

University of Newcastle,

Newcastle,

New South Wales 2308,

Australia;

Department of Mathematics,

University of New England,

Armi dale,

New South Wales 2351,

Australia;

Department of Mathematics,

Dalhousie University,

Hal ifax,

Nova Scotia B3H 4H8,

Canada. 